\title{
Rare Presentation of Toxoplasma Pneumonitis in the Absence of Neurological Symptoms in an AIDS Patient and Use of Next-Generation Sequencing for Diagnosis
}

\author{
Moni Roy ${ }^{1}$, Nikhut Siddique ${ }^{2}$, Bindu Bathina ${ }^{1}$, Sharjeel Ahmad ${ }^{3}$ \\ ${ }^{1}$ Department of Internal Medicine, University of Illinois College of Medicine, Peoria, Illinois AND OSF Saint Francis Medical Center, Peoria, Illinois \\ ${ }^{2}$ Department of Internal Medicine, University of Illinois College of Medicine, Peoria, Illinois, USA \\ ${ }^{3}$ Department of Internal Medicine, Section of Infectious Diseases, University of Illinois College of Medicine, Peoria, Illinois, USA
}

Received: 01/07/2020

Accepted: 07/07/2020

Published: 04/09/2020

How to cite this article: Roy M, Siddique N, Bathina B, Ahmad S. Rare presentation of toxoplasma pneumonitis in the absence of neurological symptoms in an AIDS patient and use of next-generation sequencing for diagnosis. EJCRIM 2020;7: doi:10.12890/2020_001862.

Conflicts of Interests: The Authors declare that there are no competing interests.

This article is licensed under a Commons Attribution Non-Commercial 4.0 License

\section{ABSTRACT}

Toxoplasma gondii is a known cause of encephalitis in human immunodeficiency virus/acquired immunodeficiency syndrome (HIV/ AIDS) patients. Toxoplasma pneumonitis is a manifestation of extracerebral toxoplasmosis and can be clinically indistinguishable from other opportunistic infections including Pneumocystis jirovecii pneumonia (PJP) and miliary tuberculosis. In this case report, Toxoplasma pneumonitis and disseminated toxoplasmosis was diagnosed using next-generation sequencing (NGS) and polymerase chain reaction (PCR) assessment. NGS can detect microbial cell-free DNA (cfDNA) circulating in the plasma of over 1,000 pathogens. This case is a rare presentation of Toxoplasma pneumonitis in the absence of neurological symptoms and we discuss the use of NGS of microbial cfDNA and PCR tests that may be utilized for the timely diagnosis of such challenging cases.

\section{LEARNING POINTS}

- Next-generation sequencing can help make a correct diagnosis and detect culture-negative opportunistic infections.

- Recognition of Toxoplasma pneumonitis as a rare presentation of disseminated toxoplasmosis.

- In cases of Toxoplasma pneumonitis, brain imaging should be conducted to rule out CNS involvement even in the absence of neurological symptoms.

\section{KEYWORDS}

Disseminated toxoplasmosis, Toxoplasma pneumonitis, next-generation sequencing, HIV/AIDS

\section{CASE DESCRIPTION}

A 39-year-old male immigrant from the Democratic Republic of the Congo presented with 5 days of fever, nausea, vomiting, productive cough and non-specific abdominal pain. He endorsed weight loss and fatigue. He denied any recent sick contacts. He denied phonophobia, photophobia, neck stiffness or headaches. His social history was unremarkable for smoking, alcohol or illicit drug use. He immigrated to the USA 12 days prior to presentation. He was sexually active with 2 females in the past year. His family history was non-contributory. The physical examination was remarkable for an ill-appearing, cachectic male with tachypnoea. Cardiac, neurological, integument and lymphatic examinations were unremarkable. 


\section{METHODS AND PROCEDURES}

A chest $\mathrm{x}$-ray showed multinodular changes in both lungs (Fig. 1). Computed tomography of the chest showed bilateral scattered miliary lesions with significant consolidations in the bases (Fig. 2). One day after admission, he admitted to a known history of HIV infection diagnosed a year previously. He denied any prior antiretroviral therapy. Severe Pneumocystis jirovecii pneumonia (PJP) or tuberculosis (TB) in an immunocompromised patient were strongly suspected. The white blood cell count was $8 \times 10^{3} / \mathrm{mcl}$. HIV antibody testing was positive for HIV-1. The absolute CD4 count was 6 and the HIV-1 viral load was 332,909 copies/ml. Influenza antigen testing was negative. The TB interferon-gamma release assay (IGRA) was indeterminate, likely secondary to a poor immune response. Acid-fast bacilli (AFB) stain and MTB culture was negative for the 3 sputum samples obtained. TB PCR testing performed on these samples was negative as well. The patient's respiratory status worsened, and he was intubated. He underwent bronchoscopy and bronchoalveolar lavage (BAL) testing was negative for AFB, fungi or bacteria on culture. BAL silver staining and testing for Histoplasma antigen, Aspergillus antigen, Legionella antigen, and varicella-zoster PCR assessment were all negative. Urine testing for Streptococcus pneumoniae antigen and Legionella antigen, along with gonococcal and Chlamydia PCR assessment, was negative. Serum syphilis IgG and Cryptococcus antigen testing was negative as well. Cytomegalovirus IgG testing was positive. Blood cultures for regular bacteria, fungi and AFB showed no growth.

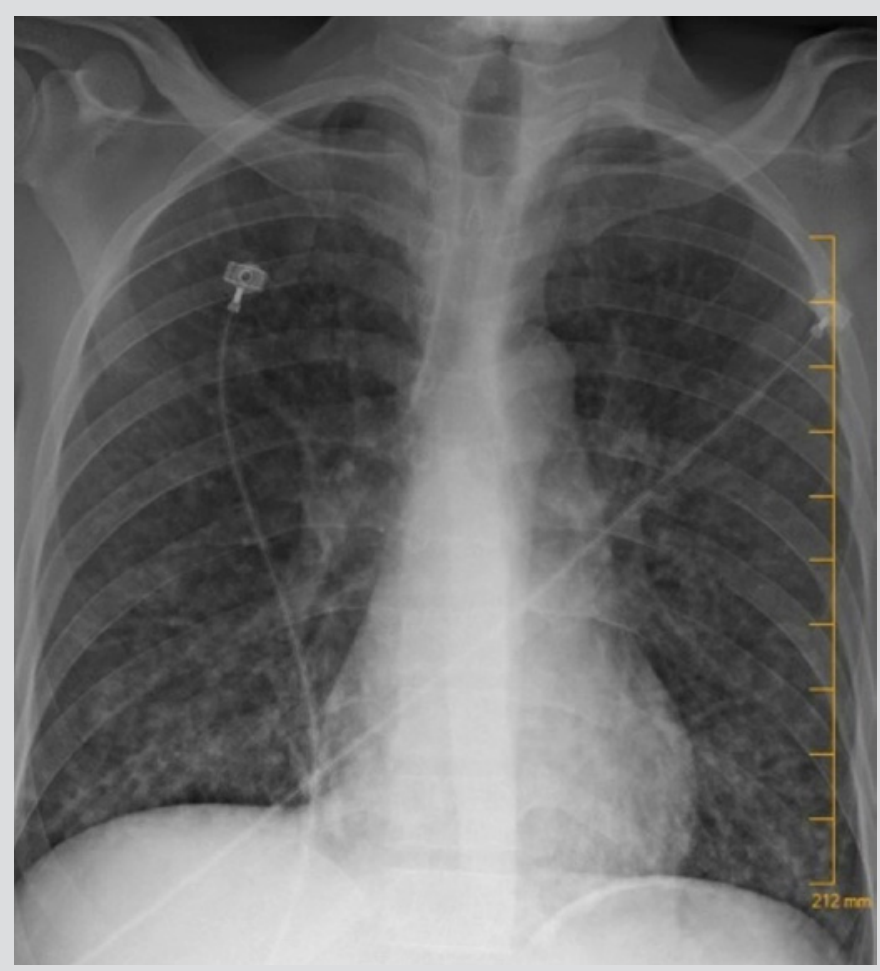

Figure 1. Chest $x$-ray with multinodular changes in both lungs

A blood sample was sent for next-generation sequencing (NGS) of microbial cell-free DNA (cfDNA) ("Karius Test", Karius Inc., Redwood City, CA, USA) on Day 11 of hospitalization, which showed high levels of Toxoplasma gondii. Toxoplasma lgG testing returned positive and IgM was negative. Following these results, Giemsa staining was performed on a BAL sample, which was negative. The BAL sample was then sent for Toxoplasma PCR assessment to 2 different reference laboratories (University of Washington Medical Center, Seattle, WA, USA and Mayo Clinic Laboratories, Rochester, MN, USA), which was found to be positive. Serum Toxoplasma PCR assessment (Mayo Clinic Laboratories, Rochester, MN, USA) was found to be positive as well. Even though the patient reported no neurological symptoms, an MRI brain scan was carried out and revealed multiple hyperattenuating infratentorial and supratentorial lesions at varying stages with ring enhancement, highly suggestive of haematogenously disseminated toxoplasmosis (Fig. 3).

Initially on presentation, the patient was started on vancomycin and piperacillin-tazobactam. Given his low CD4 count, he was started on trimethoprim-sulfamethoxazole empirically for Pneumocystis jirovecii and rifampin, isoniazid, pyrazinamide and ethambutol were added due to concerns of TB. Vancomycin was discontinued after the patient's methicillin-resistant Staphylococcus aureus (MRSA) nares swab was negative. 

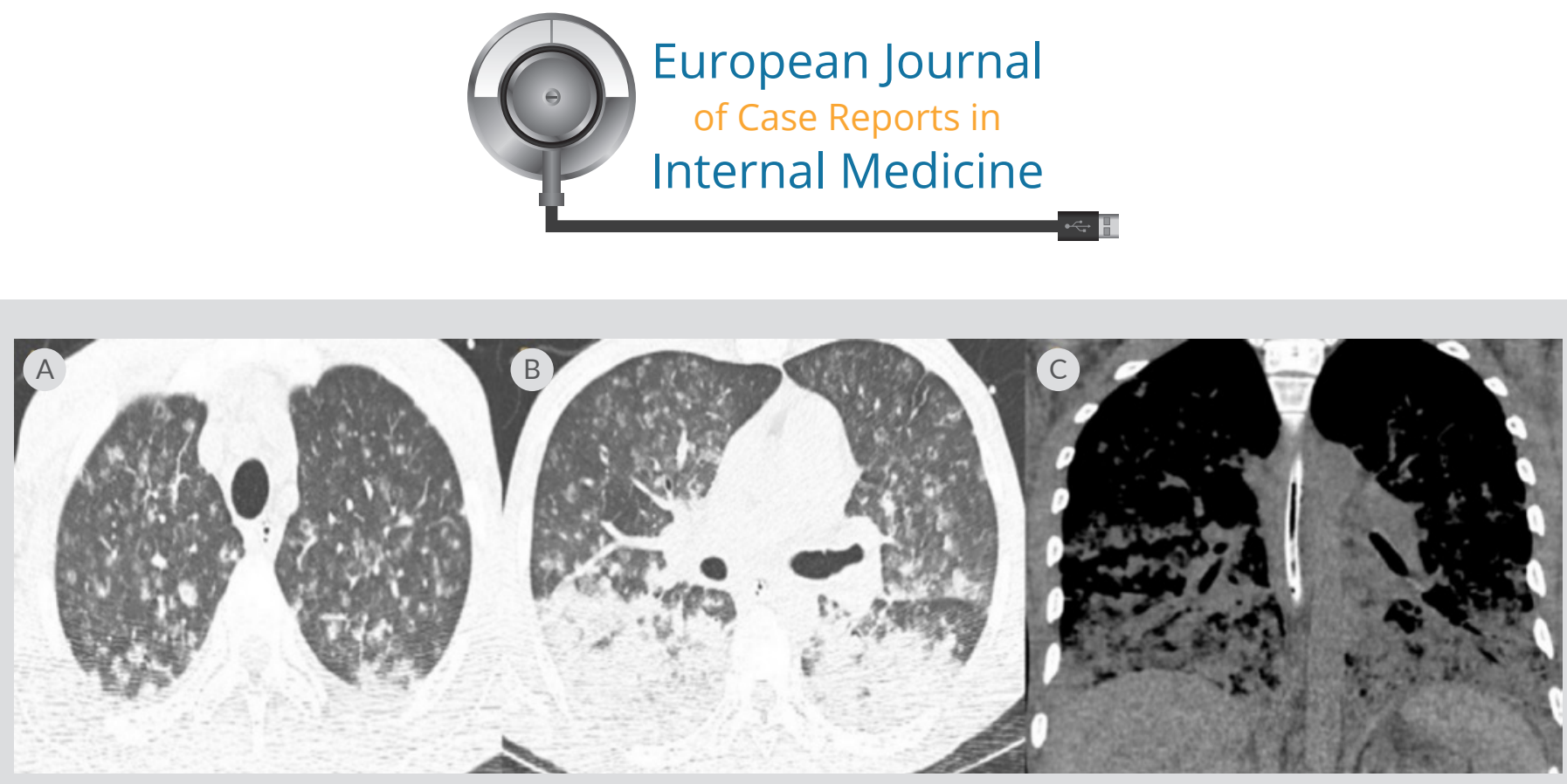

Figure 2. (A) CT chest axial lung window showing miliary changes in both lungs; (B) CT chest axial lung window showing several nodules with developing central cavitation and bilateral consolidation; (C) coronal window showing bibasilar airspace disease with confluent consolidation throughout the lung bases

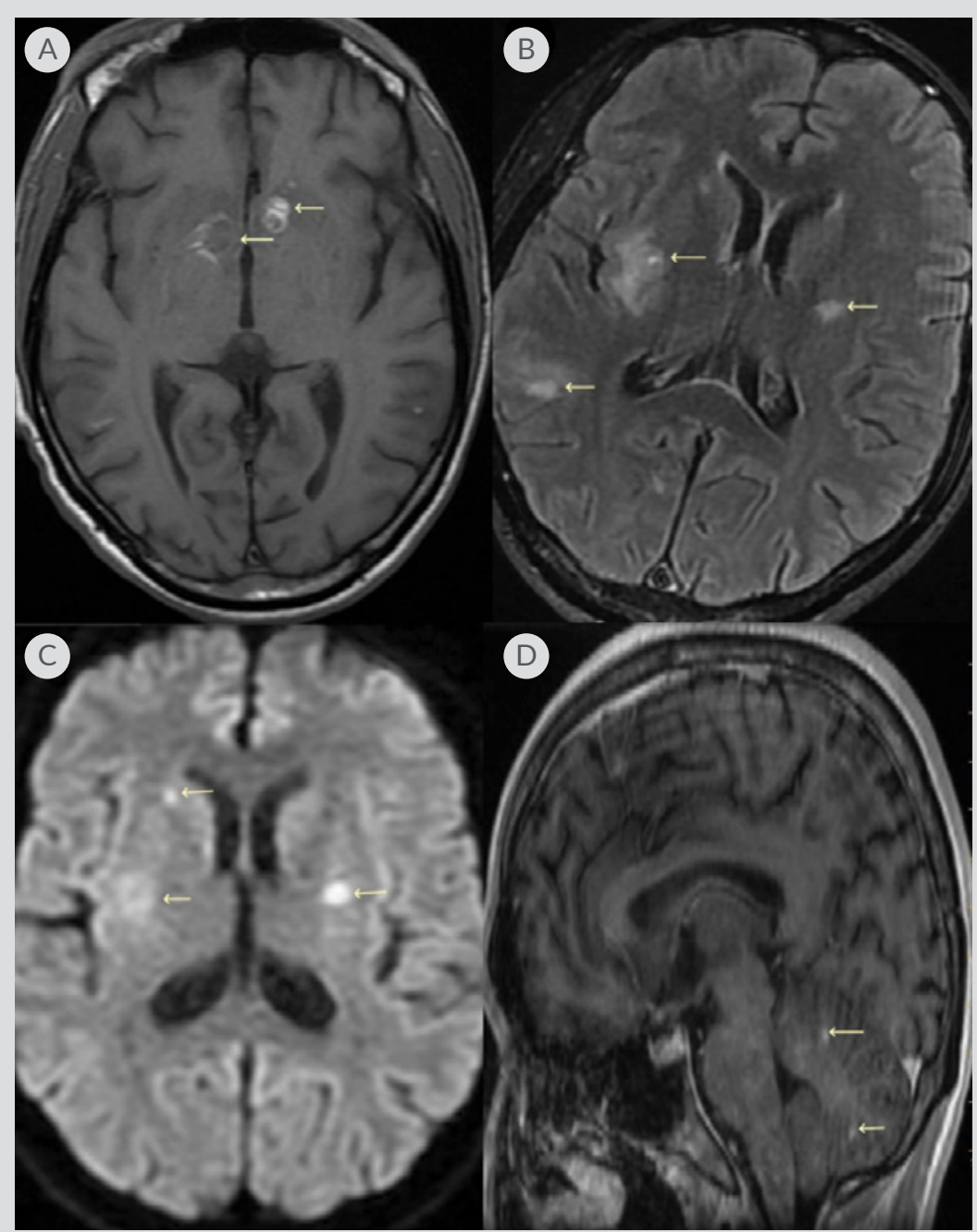

Figure 3. (A) T1 axial MRI brain image showing bilateral lesions with hyperintensity peripherally and low signal centrally; (B) T2 FLAIR MRI brain image with multiple lesions and findings suggestive of haematogenously disseminated toxoplasmosis; (C) diffusion-weighted axial brain MRI with lesions noted; (D) sagittal MRI showing lesions in the cerebellum. Leftward arrows in all images show multiple brain lesions at different stages 
Anti-TB therapy was discontinued after sputum MTB PCR testing and AFB smears turned negative. Highly active antiretroviral therapy (HAART) for HIV was initially held off due to the high risk of development of severe immune reconstitution inflammatory syndrome (IRIS) as the patient had clinical evidence of a high disease burden.

After Toxoplasma IgG positivity, confirmed with positive PCR and NGS testing, the patient was started on pyrimethamine and sulfadiazine. Piperacillin-tazobactam was discontinued. He continued a 6-week course of sulfadiazine 1,000 mg every 6 hours PO, pyrimethamine 50 mg once daily PO and leucovorin $25 \mathrm{mg}$ once daily PO, with planned maintenance therapy with sulfadiazine 1,000 mg every 12 hours PO, pyrimethamine $25 \mathrm{mg}$ once daily PO and leucovorin $10 \mathrm{mg}$ once daily PO until the CD4 count >200 for 6 months. Two weeks into treatment, the patient was started on antiretroviral therapy for HIV with bictegravir-emtricitabine-tenofovir alafenamide 50-200-25 mg once daily. After appropriate treatment for toxoplasmosis was initiated, our patient improved significantly. Three weeks after starting antiretroviral therapy, his HIV viral load dramatically decreased to 2,986 copies/ml and his absolute CD4 count improved to 66 .

\section{DISCUSSION}

Toxoplasma is an intracellular parasite and in immunocompromised hosts it can reactivate to present with symptoms of encephalitis. In immunocompromised patients, multinodular lung lesions raise suspicion for opportunistic infections, most commonly PJP or TB. Toxoplasma pneumonitis in the absence of CNS symptoms is a rare presentation and a high index of suspicion is required to make the correct diagnosis ${ }^{[1]}$. Very few cases of pulmonary toxoplasmosis in the absence of neurological symptoms have been reported ${ }^{[2,3]}$. A retrospective study of 13 patients with HIV and Toxoplasma pneumonia showed that most cases had very low CD4 counts and coexisting non-Hodgkin's lymphoma ${ }^{[4]}$. The diagnosis of isolated Toxoplasma pneumonitis is a challenge as imaging findings are non-specific and conventional diagnostic methods such as culture and serology lack sensitivity ${ }^{[5]}$. NGS can detect cfDNA of DNA viruses, fungi and protozoa in plasma, both in cases of localized or disseminated infection ${ }^{[6]}$. Due to the ability of NGS to detect active as well as latent infections, the results should be interpreted in conjunction with coexisting clinical symptoms, radiological and other laboratory findings as the reported microorganism may or may not be the cause of infection. As per our literature review, the use of NGS in the diagnosis of toxoplasmosis in humans has not been extensively described. Hu et al. recently reported the use of NGS in the diagnosis of Toxoplasma encephalitis ${ }^{[7]}$. Timely diagnosis of Toxoplasma pneumonia in the absence of neurological symptoms is even more challenging. We believe there is a role for NGS in detecting this pathogen early in the course of infection. We also suggest work-up for disseminated infection by carrying out brain MRI to look for CNS involvement, even in the absence of symptoms. Understanding the potential of newer diagnostic modalities such as NGS is of immense value, and as medical technology continues to advance, we may be able to diagnose opportunistic infections more definitively and efficiently.

\section{REFERENCES}

1. Mariuz P, Bosler EM, Luft BJ. Toxoplasma pneumonia. Semin Respir Infect 1997;12(1):40-43.

2. Schnapp LM, Geaghan SM, Campagna A, Fahy J, Steiger D, Ng V, et al. Toxoplasma gondii pneumonitis in patients infected with the human immunodeficiency virus. Arch Intern Med 1992;152(5):1073-1077.

3. Bergin C, Murphy M, Lyons D, Gaffney E, Mulcahy FM. Toxoplasma pneumonitis: fatal presentation of disseminated toxoplasmosis in a patient with AIDS. Eur Respir J 1992;5(8):1018-1020.

4. Oksenhendler E, Cadranel J, Sarfati C, Katlama C, Datry A, Marche C, et al. Toxoplasma gondii pneumonia in patients with the acquired immunodeficiency syndrome. Am J Med $1990 ; 88(5 \mathrm{~N}): 18 \mathrm{~N}-21 \mathrm{~N}$.

5. Murat JB, Hidalgo HF, Brenier-Pinchart MP, Pelloux H. Human toxoplasmosis: which biological diagnostic tests are best suited to which clinical situations? Expert Rev Anti Infect Ther 2013;11(9):943-956.

6. Hong DK, Blauwkamp TA, Kertesz M, Bercovici S, Truong C, Banaei N. Liquid biopsy for infectious diseases: sequencing of cell-free plasma to detect pathogen DNA in patients with invasive fungal disease. Diagn Microbiol Infect Dis 2018; 92(3):210-213.

7. Hu Z, Weng X, Xu C, Lin Y, Cheng C, Wei H, et al. Metagenomic next-generation sequencing as a diagnostic tool for toxoplasmic encephalitis. Ann Clin Microbiol Antimicrob 2018;17:45. 